\title{
$N 90-22958$
}

\section{INTERACTIONS OF FORM AND ORIENTATION}

\author{
Horst Mittelstaedt \\ Max-Planck-Institut fur Verhaltensphysiologie, D-8130 Seewiesen \\ Bundes Republik Deutschland
}

\section{EFFECT OF ORIENTATION OF PERCEPTION OF FORM}

It is well know that the orientation of an optical pattern relative to egocentric or extraneous references affects its figural quality, that is, alters its perceived form and concomitantly delays or quickens its identification (Rock 1973). A square presented in the frontal plane to an upright person (S), for instance, changes from a "box" to a "diamond" when it is rotated with respect to the $S$ 's median plane by $45^{\circ}$. This angle, that is, the angle between the orientations of the pattern in which the two apparent figures ("Gestalten") attain a summit of purity and distinctness, will be called the "figural disparity" of the pattern. If, as in this case, the $S$ is upright, the retinal meridian and the subjective vertical (SV) are both in the viewer's median plane. The question arises with respect to which of these orientation references the two figures are identified. The answer may be found when the pattern and the $S$ are oriented in such a way that the projections of the retinal meridian and the SV into the plane of the pattern diverge by the pattern's figural disparity or its periodic multiples; that is, in the case of a square by $45^{\circ}$ or $135^{\circ}$, respectively. Similarly, which reference determines whether an equilateral triangle is seen as a "pyramid" or a "traffic warning sign" may be revealed at a divergence of SV and retinal meridian of $60^{\circ}$ or $180^{\circ}$, respectively. It is generally found that for head roll tilts $(\rho)$ and figural disparities of up to $90^{\circ}$, the figure whose axis coincides with the SV is seen. At head tilts of $\rho=180^{\circ}$, however, the retinal reference dominates, as a rule independently of the figural disparity (for reviews, see Rock 1973 and Howard 1982).

\section{EFFECT OF FORM ON PERCEPTION OF ORIENTATION}

Clearly, then, orientation may determine apparent form. But conversely, form may also influence apparent orientation. This is explicitly true in the case of the SV (for review, see Bischof 1974; for the recent state, see Wenderoth 1976; Mittelstaedt 1986).

As shown in Fig. 1, our method is to project the pattern within a circular frame (of $16^{\circ}, 35^{\circ}$, or $80^{\circ}$ visual angle) into a tilted planetarium cupola $(\phi=9.1 \mathrm{~m})$ in 24 stationary orientations presented to the $S$ in a pseudo-random sequence. The $S$, lying on her side, indicates her SV by means of a rotatable luminous line, which is projected onto the cupola such that its center of rotation coincides with the center of the pattern's circular frame and the S's visual axis.

The effect of the pattern on the SV turns out to be a rather involved function of the orientation of the pattern. This relation becomes clear, however, if we assume that the luminous line is eventually oriented such that the effect of the pattern is opposite and equal to the nonvisual effect on the SV, exerted mainly by the vestibular system. Both effects are then expected to be functions of the difference between the angle $\beta$ at which the luminous line is set with the pattern present and the angle $\beta_{\mathrm{g}}$ at which it is found in the absence of visual cues. For the nonvisual effect, fortunately, this function may be computed according to an extant theory (Mittelstaedt 1983a,b): the SV is influenced not only by information about head tilt, but also by intrinsic parameters which are independent of head tilt, notably the "idiotropic vector" (M). Presumably by addition of constant endogeneous discharges to the saccular output, it leads to a perpetual shift of the SV into the 
direction of the S's long axis and hence causes the phenomenon which is well known as the Aubert phenomenon. At first approximation, this relation may be represented by a vector diagram (Fig. 2): In the absence of visual cues, the SV is perceived in the direction of the resultant $\mathrm{R}$ of the otolithic vector $G$ and the idiotropic vector $M$.

In our case, since $\rho=90^{\circ}$, the nonvisual effect $g$ becomes a particularly simple function $\beta-\beta_{\mathrm{g}}$, namely,

$$
\begin{aligned}
g= & \sqrt{G^{2}+M^{2}} \sin \left(\beta-\beta_{g}\right)=\sqrt{G^{2}+M^{2}} \sin \left(\beta-\operatorname{arccotan} \frac{M}{G}\right) \\
& =M \sin \beta-G \cos \beta
\end{aligned}
$$

Because of the normalization of the vestibular information (which is inferred from effects of centrifugation), $g$ may be computed with $G=1$ and $M=\operatorname{cotan} \beta_{g}$. Hence the unknown visual effect on the SV may be determined if the known quantity $\mathrm{g}$ is plotted as a function of the angle on which effect of the pattern depends. There seem to be only two possible candidates: the angle $\vartheta$ between the pattern's main axis and the S's long axis, or the angle $\beta-\vartheta$ between the former and the present direction $\beta$ of the SV.

Figure 3 shows plots of this latter function (named SV-function) engendered in three Ss by a color slide of the house of Fig. 1. It turns out that the visual effect is zero, that is, does not change the SV $\left(\beta=\beta_{g}\right)$ if and only if $\beta-\vartheta$ is zero, rather than when $\vartheta$ is zero. Hence its magnitude must be a function of the former angle. We may envisage the $S V$ as being at equilibrium between two tendencies ("torques"), (1) the gravito-idiotropic torque $\mathrm{g}$, trying to pull it toward $\beta-\beta_{g}=0$, (2) the other, the visual torque $v$, trying to pull it toward $\beta-\vartheta=0$ (see Fig. 2). Generally, the visual torque exerted on the SV by a pattern turns out to be an antisymmetrical periodic function composed of the sine of $(\beta-\vartheta)$ and the sine of the angle's multiples. Hence it may be simply and fully characterized by the amplitudes $V_{n}$ of these sine components, to be called "(circular) harmonics" of the respective SV function. With the picture of the house of Fig. 1 as well as with other photographed scenes, the first circular harmonic is generally found to vary greatly inter- as well as intrapersonnally. By contrast, the second and fourth harmonics vary but moderately (within an order of magnitude) between Ss, and are rather constant intrapersonnally for a given pattern. ${ }^{1}$ The formal difference is supposed to be due to a difference in the underlying information processing. The first harmonic expresses the effect of the picture's bottom-to-top polarity, that is, of those cues for the vertical which may be inferred from its normal orientation to gravity. The recognition of what is the top must probably be learned through personal experience, and its effect is hence expected to vary with individual visual proficiency. The even-number harmonics, by contrast, are presumably based on invariant structures of the visual system, possibly by a weighting process, from the "simple cells" of the visual cortex (Mittelstaedt 1986).

This is highlighted by the following experimental series. If orthogonal lines are presented as a pattern, the resulting SV-function contains only circular harmonics which are multiples of four.

${ }^{1}$ All circular harmonics higher than the fourth, except for the eighth, which is sometimes found to be just above noise level, are insignificant or zero. With the sampling used, the amplitudes of the first four harmonics were about the same irrespective of whether the Fourier analysis was made with the equidistant sampling of plots over $\vartheta$ or with the, necessarily, scattered sampling of plots over $\beta-\vartheta$ as in Fig. 3. 
The fourth usually is then the largest and is positive; that is, at its null-crossings with positive slope the SV coincides (is in phase) with the direction of the lines (Fig. 4).

If a pictograph of a human figure is presented which consists of uniformly oriented lines (Fig. 5; "star man") or random dashes, the first harmonic is in phase with star man's long axis and hence is positive.

What will happen if the pictograph of a human figure is presented which consists, as in Fig. 6 ("diamond man"), exclusively of lines that are oriented at $45^{\circ}$ with respect to the figure's long axis? As a matter of fact, the two figural components are superimposed: the first harmonic is in phase and hence positive; the fourth is in counterphase and hence negative, neither "taking notice" of the other (Fig. 7).

Evidently, the result falsifies the hypothesis (Bischof and Scheerer 1970) that the CNS first computes a "resultant visual vertical" of the picture and subsequently forms an antisymmetrical periodic function in phase with this resultant. For then, the resultant would either coincide with the long axis of diamond man and hence the fourth harmonic would be positive, or (rather unlikely though) the resultant would coincide with one of the line directions and hence the first harmonic would be in phase with that line (or would be missing). Instead, the first harmonic results from a processing which is determined by the bottom-to-top polarity of the picture independently of its unpolarized axial features. At the same time, the even-number harmonics are determined by the pattern's unpolarized axial features independently, at least with respect to phase, of its bottom-totop polarity.

\section{INTERRELATIONS BETWEEN THE DETERMINANTS OF APPARENT VERTICAL AND OF FORM PERCEPTION}

It shall now be examined whether, by means of the comprehensive mathematical theory of the SV, understanding the effect of perceived form on the SV may help in understanding the effect of the SV on form perception mentioned earlier.

First, the theory does indeed offer a good reason why the influence of the SV on the perception of form should decrease with an increasing tilt angle of the $S$. The effect of the otolithic output than decreases (besides due to comparatively small deviations from a linear response to shear) as a consequence of the addition of the idiotropic vector. Its amount is an idiosyncratic constant averaging around $50 \%$ of that of $G$. The magnitude of the resultant $R$ of the idiotropic vector $M$ and the gravity vector $\mathrm{G}$ may be approximated as

$$
R=\sqrt{G^{2}+M^{2}+2 G M \cos \rho}
$$

Evidently, $R$ must decrease with increasing angle of tilt $\rho$, and so will its relative influence when competing with visual cues!

Second, the theory may open a way to assess the relative strength of the factors that influence form perception. The influence of visual patterns on the SV is not independent of the angle of tilt (Bischof and Scheerer 1970). This effect may be quantitatively described by weighting the visual torque $v$ with the sum of the squared saccular and utricular (roll) components (for details see 
Mittelstaedt 1986). Hence the effect of the visual torque is maximal at a roll tilt between 60 and $90^{\circ}$ and declines toward the upright as well as toward the inverted posture. As a result, at small roll tilts of the $S$, the nonvisual torque $g$ may, under certain conditions, be larger than the visual torque, about equal to the latter around $\rho=90^{\circ}$, but much smaller than the visual torque when the $S$ is inverted $\left(\rho=180^{\circ}\right)$. Which component will determine which form is perceived under which angle of divergence may be predictable, if the relative weights of the nonvisual and visual components in the determination of the SV would be correlated with the relative weights of the two reference systems in the perception of form.

\section{SUPPRESSION OR ADDITIVE SUPERPOSITION}

However, the underlying information-processing systems may be fundamentally different in the two cases. Evidently, additive superposition suffices to explain the interaction of the components in the case of the SV. But in their influence on form perception, a decision in case of conflict appears to be called for, and hence to necessitate a nonlinear interaction in that one of the competitors is suppressed.

This we have tested by using the well-known ambiguous figure of Fig. 8 . It is seen, by an upright $S$, as a "princess" $\mathrm{P}$ or a "witch" $\mathrm{W}$ when the long axis of $\mathrm{P}$ is aligned or reversed with respect to the $S$ 's long axis.

If the $S$ is tilted by $180^{\circ}$ relative to gravity $\left(\rho=180^{\circ}\right)$ the retinal reference determines the perception, as is generally found in comparable cases. The crucial situation arises when the $S$ views the figure while lying on the $\left(\rho=90^{\circ}\right)$. In this position the figure was presented at various angles $v$ with respect to the $S$ 's long axis, and the $S$ was instructed to report whether the witch or the princess appeared more distinctly. In order to determine the point of transition between the two phenomena, their distinctness was scaled by the Ss in seven steps, which are condensed in Fig. 9 into five (exclusively $\mathrm{P}$; preponderantly $\mathrm{P}$; ambiguous; preponderantly $\mathrm{W}$; exclusively $\mathrm{W}$ ).

Two Ss, who were well versed in psychophysical tests were chosen. In addition their SV in the absence of visual cues and their ocular counterroll at $\rho=90^{\circ}$ were determined and were found as shown in Fig. 9. Clearly, in both Ss, the midline between the transition zones neither coincides with the SV nor with the retinal meridian, but assumes an intermediate direction between these two. Hence even in their influence on form perception the gravito-idiotropic and the visual effects may combine vectorially rather than suppress one another.

It is advisable, then, to reexamine those instances where an exclusive decision between the two references is found. As mentioned earlier, this happens regularly, when $\mathrm{S}$ and pattern are placed such that the $S V$ and the retinal meridian diverge by the figural disparity angle. Now let the "salience" s (die "PRAEGNANZ") of a figure (X) vary as a symmetrical periodic function of its deviation from the respective reference such that

$$
s_{x}=\sum_{n=0}^{\max } E_{x n} \cos n \vartheta_{x}^{\prime}+\sum_{n=0}^{\max } V_{x n} \cos n\left(\beta-\vartheta_{x}^{\prime}\right)
$$

where $\vartheta$ is the angle between the figure's main axis and the retinal meridian, $\beta^{\prime}$ is the angle between the SV and the retinal meridian, and $E_{x n^{\prime}}, V_{x n}$ are the amplitudes of the figure's circular 
harmonics weighted (as suggested in section 3) by the retinal $\left(E_{x n}\right)$ and the SV reference systems $\left(V_{x n}\right)$, respectively. The central nervous correlate of the relative salience of figures $X, Y$ may then be determined by the difference $s_{x}-s_{y}$. In the case of princess versus witch, because $\vartheta_{\mathrm{w}}^{\prime}=\vartheta_{\mathrm{p}}^{\prime}-180^{\circ}$ and if, for the sake of simplicity, nmax is assumed to be unity, the difference becomes

$$
s_{p}-s_{w}=\left(E_{p 0}+V_{p 0}\right)-\left(E_{w 0}+V_{w 0}\right)+\left(E_{p 1}+E_{w 1}\right) \cos \vartheta_{p}^{\prime}+\left(V_{p 1}+V_{w 1}\right) \cos \left(\beta^{\prime}-\vartheta_{p}^{\prime}\right)
$$

In the upright $S\left(\rho=0, \beta^{\prime}=0\right)$, with $E_{p 0}+V_{p 0}=E_{w 0}+V_{w 0}$, this becomes

$$
s_{p}-s_{w}=\left(E_{p 1}+E_{w 1}+V_{p 1}+V_{w 1}\right) \cos \vartheta_{p}^{\prime}
$$

That is, independently of the relative weights, the princess dominates at acute angle $\vartheta_{\mathrm{p}}^{\prime}$ and the witch dominants at obtuse angles $\vartheta_{p}^{\prime}$ However, with the $S$ inverted $\left(\rho=180^{\circ}, \beta^{\prime}=180^{\circ}\right)$ :

$$
s_{p}-s_{w}=\left[\left(E_{p 1}+E_{w 1}\right)-\left(V_{p 1}+V_{w 1}\right)\right] \cos \vartheta_{p}^{\prime}
$$

Consequently, the pattern is identified exclusively according to one of the two reference systems, if their respective weighting factors differ and $\vartheta \neq 90^{\circ}$, even though the assumed processing is purely additive. The same holds for the other examples given above. In the case of the square, for instance, with $n=4$, and the $S$ tilted until $\beta^{\prime}=45^{\circ}\left(\rho \approx 45^{\circ}\right)$,

$$
s_{b(b o x)}-s_{d(d i a m o n d)}=\left[\left(E_{b 4}+E_{d 4}\right)-\left(V_{b 4}+V_{d 4}\right)\right] \cos 4 \vartheta_{b}^{\prime}
$$

This leads to a "decision" in favor of the SV-reference if - quite plausibly at that acute angle-the $V$ factors are then larger than the E factors, whereas at $\beta^{\prime}=135^{\circ}\left(135^{\circ}<\rho<180^{\circ}\right)$ they appear to be almost equal: in that position some of our Ss refused to decide about what they see! In the case of princess versus witch with the $S$ at $\rho=90^{\circ}$ and $\beta=60^{\circ}$

$$
s_{p}-s_{w}=\left(E_{p 1}+E_{w 1}\right) \cos \vartheta_{p}^{\prime}+\left(V_{p 1}+V_{w 1}\right) \cos \left(60^{\circ}-\vartheta_{p}^{\prime}\right)
$$

Hence a compromise is to be expected depending on the relative magnitudes of the weighting factors. The relative salience $\left(s_{p}-s_{w}\right)$ is then zero at $\vartheta_{\Delta p \text { zero }}^{\prime}$, and

$$
\operatorname{cotan} \vartheta_{\Delta p \text { zero }}^{\prime}=\frac{ \pm \sin 60^{\circ}}{+\left(\frac{E_{p 1}+E_{w 1}}{V_{p 1}+V_{w 1}}+\cos 60^{\circ}\right)}
$$

as is borne out by the results here and in Fig. 9.

In conclusion, the present state favors the notion that angular relations are represented and processed in the CNS by variables which are trigonometric functions of the respective angles. That the characteristics and the spatial arrangement of the otolithic receptors and of the simple cells in the visual cortex are well suited to implement this kind of coding (Mittelstaedt 1983a,b; 1986; 1988 in 
press) lends a neurophysiological backbone to the demonstrated descriptive and predictive powers of such a theory.

\section{Acknowledgments}

I am grateful to all members of my technical staff for their dedicated assistance and to Evi Fricke, Werner Haustein, Werner Mohren, Stephen R. Ellis, and Heiner Deubel for their valuable contributions to experimentation, evaluation and formulation. 


\section{REFERENCES}

Bischof, N. (1974): Optic-vestibular orientation to the vertical. In: Handbook of sensory physiology (Autrum, H. et al., eds.) 155-190 Springer, Berlin, Heidelberg, New York.

Bischof, N. and Scheerer, E. (1970): Systemanalyse der optisch-vestibularen Interaktion bei der Wahrnehmung der Vertikalen. Psychologische Forschung 34, 99-181.

Howard, I. P. (1982): Human visual orientation. Wiley \& Sons, New York, Brisbane, Toronto.

Mittelstaedt, H. (1983a): A new solution to the problem of the subjective vertical.

Naturwissenschaften 70, 272-281.

Mittelstaedt, H. (1983b): Towards understanding the flow of information between objective and subjective space. In: Neuroethology and behavioral physiology (Huber, F. and Markl, H., eds.) 382-402, Springer, Berlin, Heidelberg, New York.

Mittelstaedt, H. (1986): The subjective vertical as a function of visual and extraretinal cues. Acta Psychologica 63, 63-85.

Mittelstaedt, H. (1988 in press): The information processing structure of the subjective vertical. A cybernetic bridge between its psychophysics and its neurobiology. In: Processing structures for perception and action (Marko, H., Hauske, G., Struppler, A., eds.). Final report of the Sonderforschungsbereich "Kybernetik." Verlag Chemie, Weinheim.

Rock, I. (1973): Orientation and Form. New York: Academic Press.

Wenderoth, P. M. (1976): An analysis of the rod-and-frame illusion and its variants. In: Studies in Perception (Day, R. H., Stanley, G. S., eds.) Perth: Univ. Western Australia, Perth. 


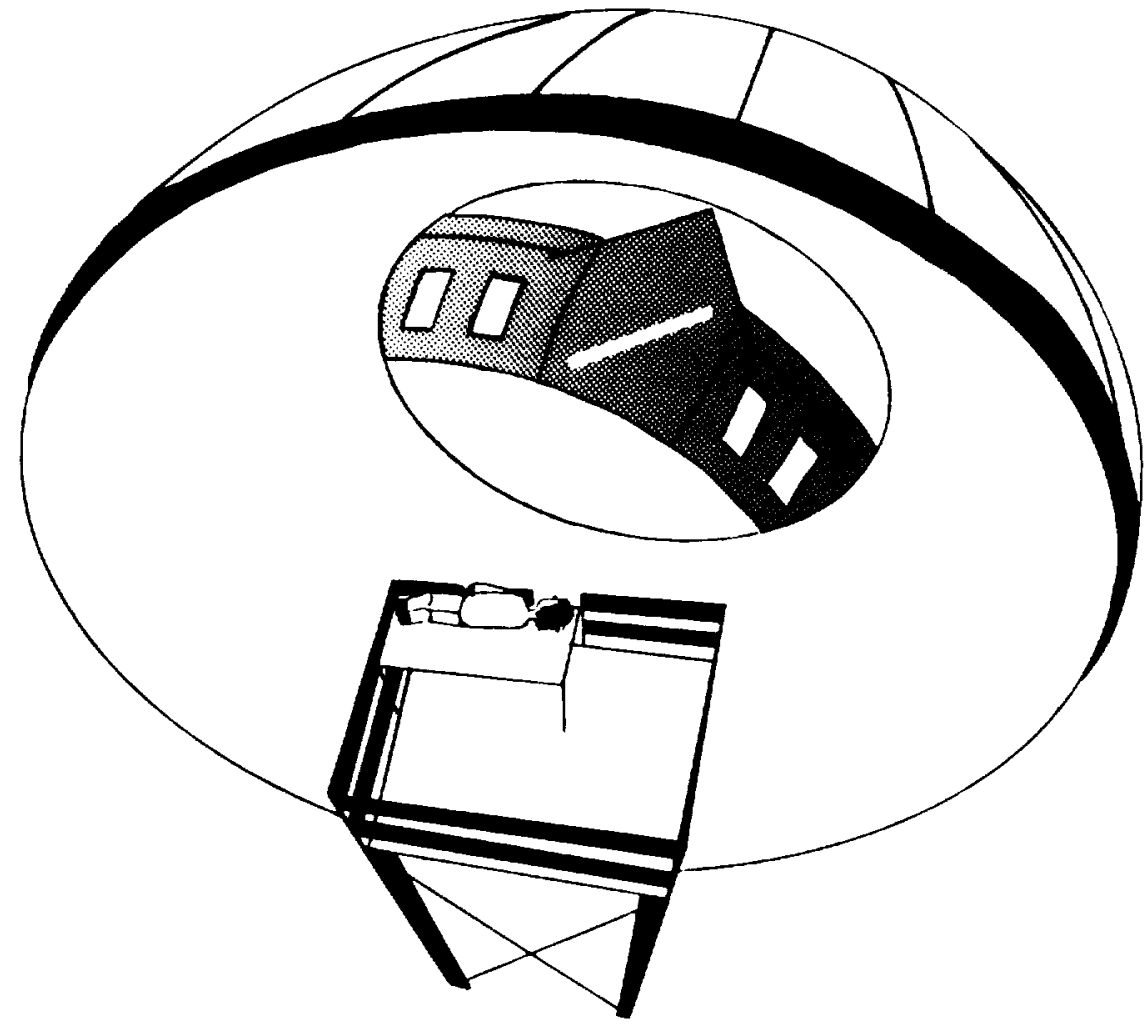

Figure 1.- Experimental setup for testing the effect of tilted images on the subjective vertical. The image is projected in a sequence of static roll tilts onto a hemispherical $(\varnothing=9.1 \mathrm{~m})$ screen in front of the subject. The $S$, lying on her side, is asked to set a projected luminous line to subjective vertical. 


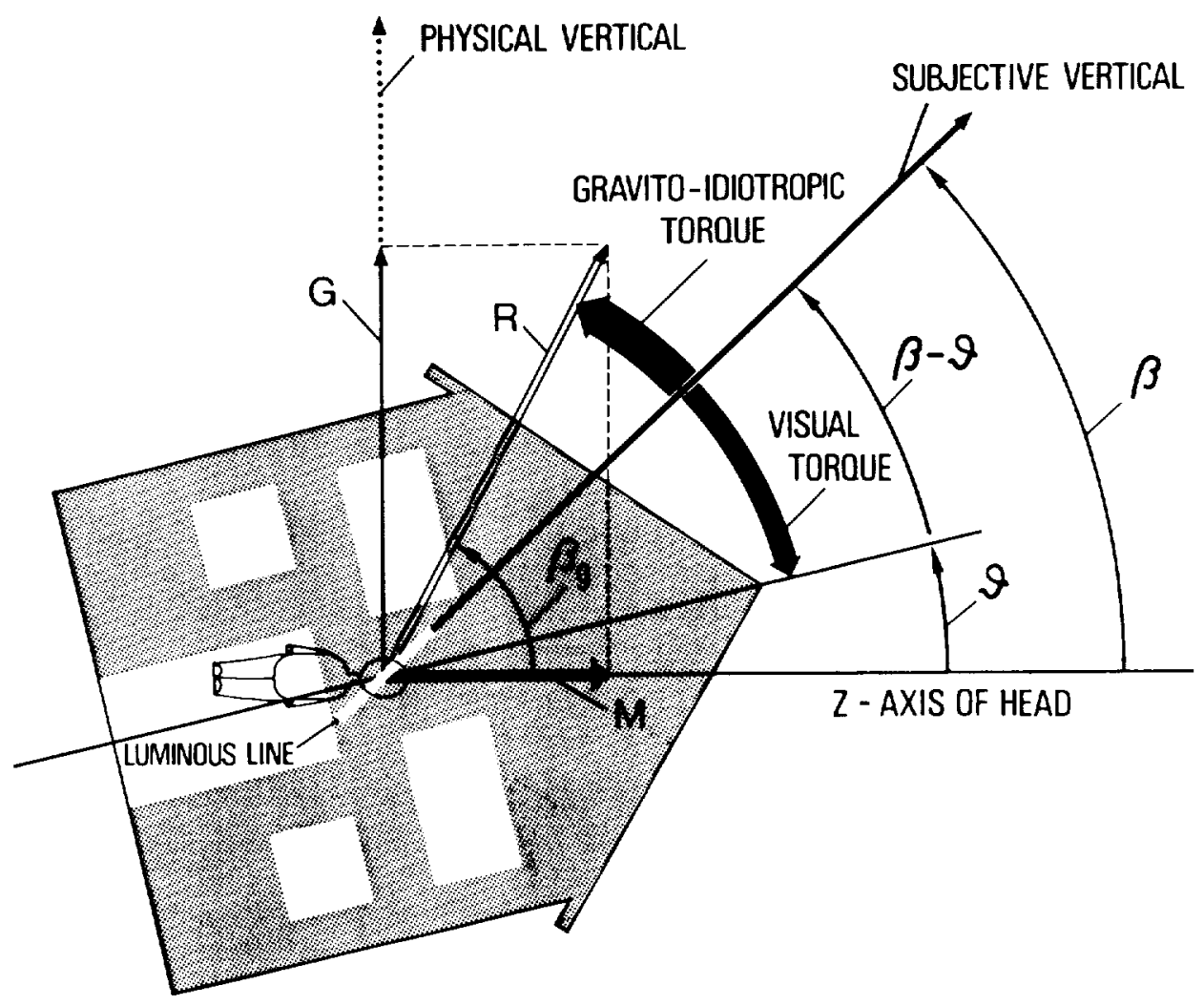

Figure 2.- Definition of critical variables and their relations to hypothetical determinants of the SV:

1) It is supposed that the visual scene (here a house) exerts an attraction effect on the SV. This "visual torque" is supposed to be a function of $\beta-\vartheta$, the angle between the main axis of the tilted image and the luminous line when set subjectively vertical; 2) This visual torque is supposedly counterbalanced by a "gravito-idiotropic torque." The latter is a function of $\beta-\beta_{g}$, the angle between the present SV and the $\beta_{g}$ the SV would have in the absence of visual cues. The latter function may be determined as

$$
g=\sqrt{G^{2}+M^{2}} \sin \left(\beta-\beta_{g}\right)=\sqrt{G^{2}+M^{2}} \sin \left(\beta-\operatorname{arccotan} \frac{M}{G}\right)=M \sin \beta-G \cos \beta
$$

with $G=1$. Hence the unknown visual torque may be quantitatively described. All angles defined with respect to (long) $\mathrm{Z}$-axis of head. 
ORIGINAL PAGE IS

OF POOR QUALITY
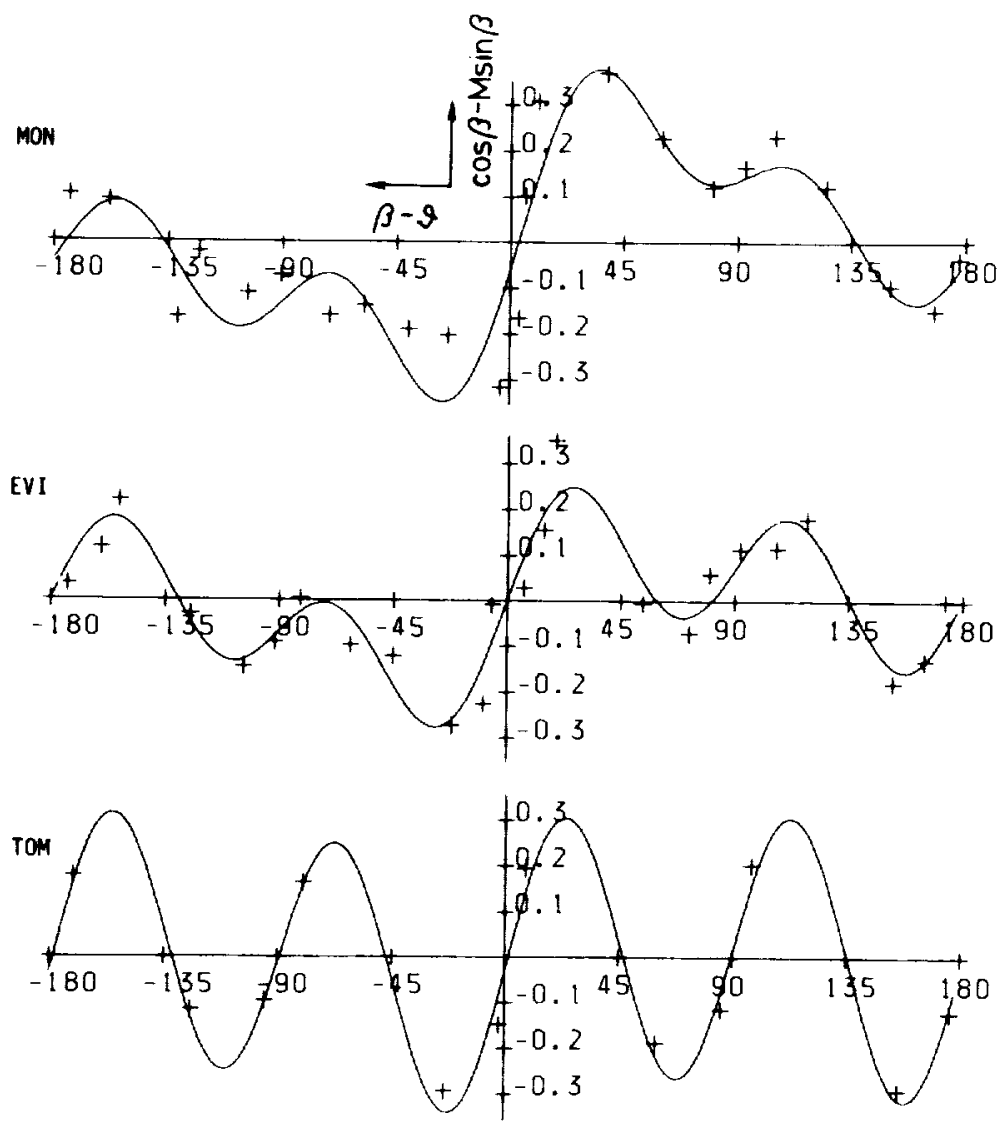

$\begin{array}{cccccc}S & M & v_{1} & v_{2} & v_{3} & V_{4} \\ \text { MON } & .68 & .178 & .137 & .043 & .122 \\ \text { EVI } & .40 & .083 & .092 & .013 & .147 \\ \text { TOM } & .19 & .011 & .036 & .001 & .293\end{array}$

Figure 3.- Effect of the same tilted scene (a house) on the SV of three Ss (MON, EVI, TOM). The gravito-idiotropic torque $-\mathrm{g}$ is plotted as a function of $\beta-\vartheta$ (see Fig. 2). Crosses: means of pairs of settings. Curves: least-square fits of summed sine functions $-g=\sum V_{n} \sin n(\beta-\vartheta)$ with amplitudes $V_{1-4}$ to the data. Note the large variation of the amplitude $V_{1}$ of the first harmonic in contrast to the moderate variation of $V_{2}$ and $V_{4}$. 

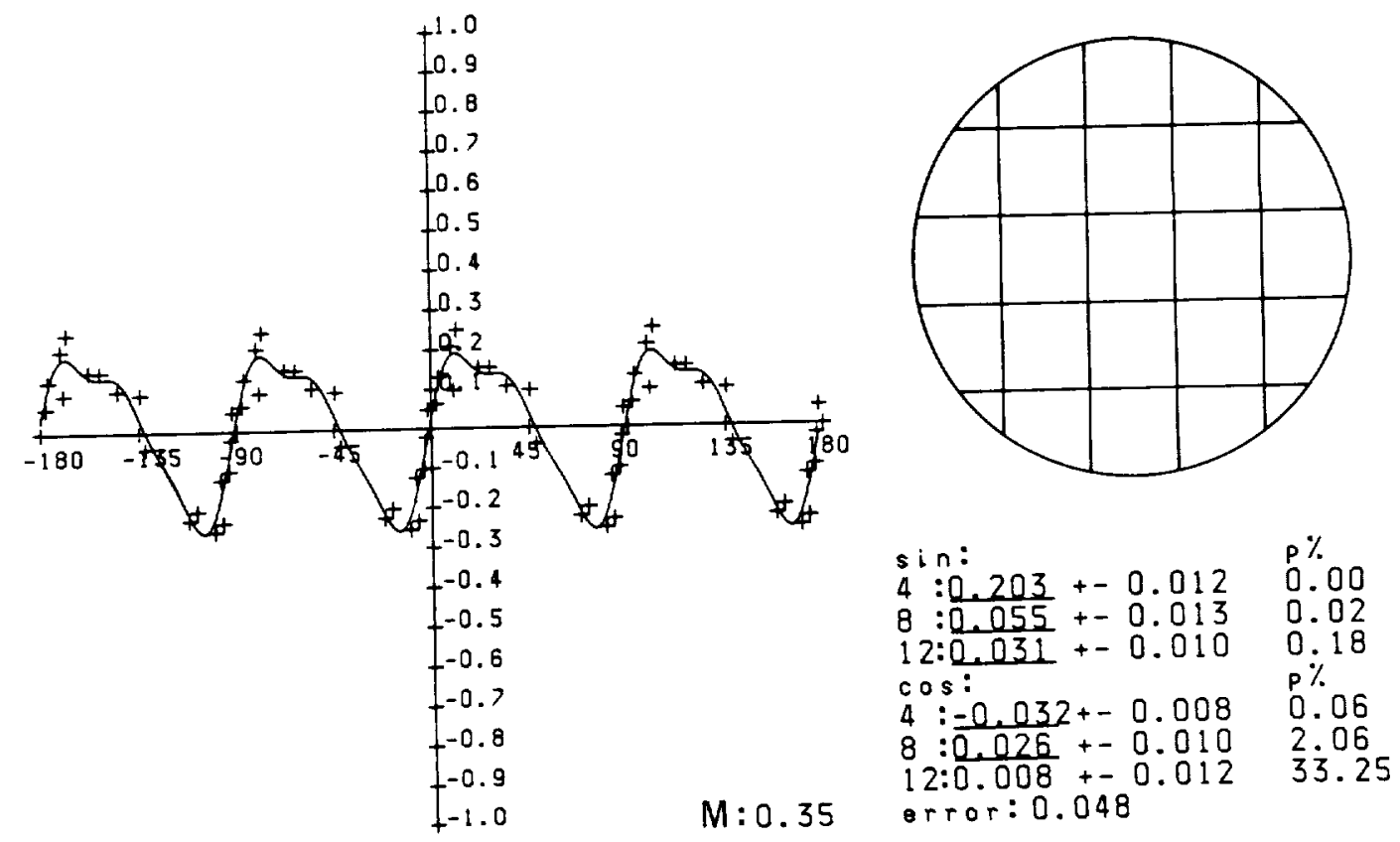

Figure 4.- Effect of pattern of squared luminous lines on SV. Method and evaluation as in Figs. 1-3. Inset gives numerical values of amplitudes (sines and cosines) of fourth, eighth, and twelfth harmonics of SV-function, their SD, and p (in \%; two-tailed). Error means square deviation of data from approximation.

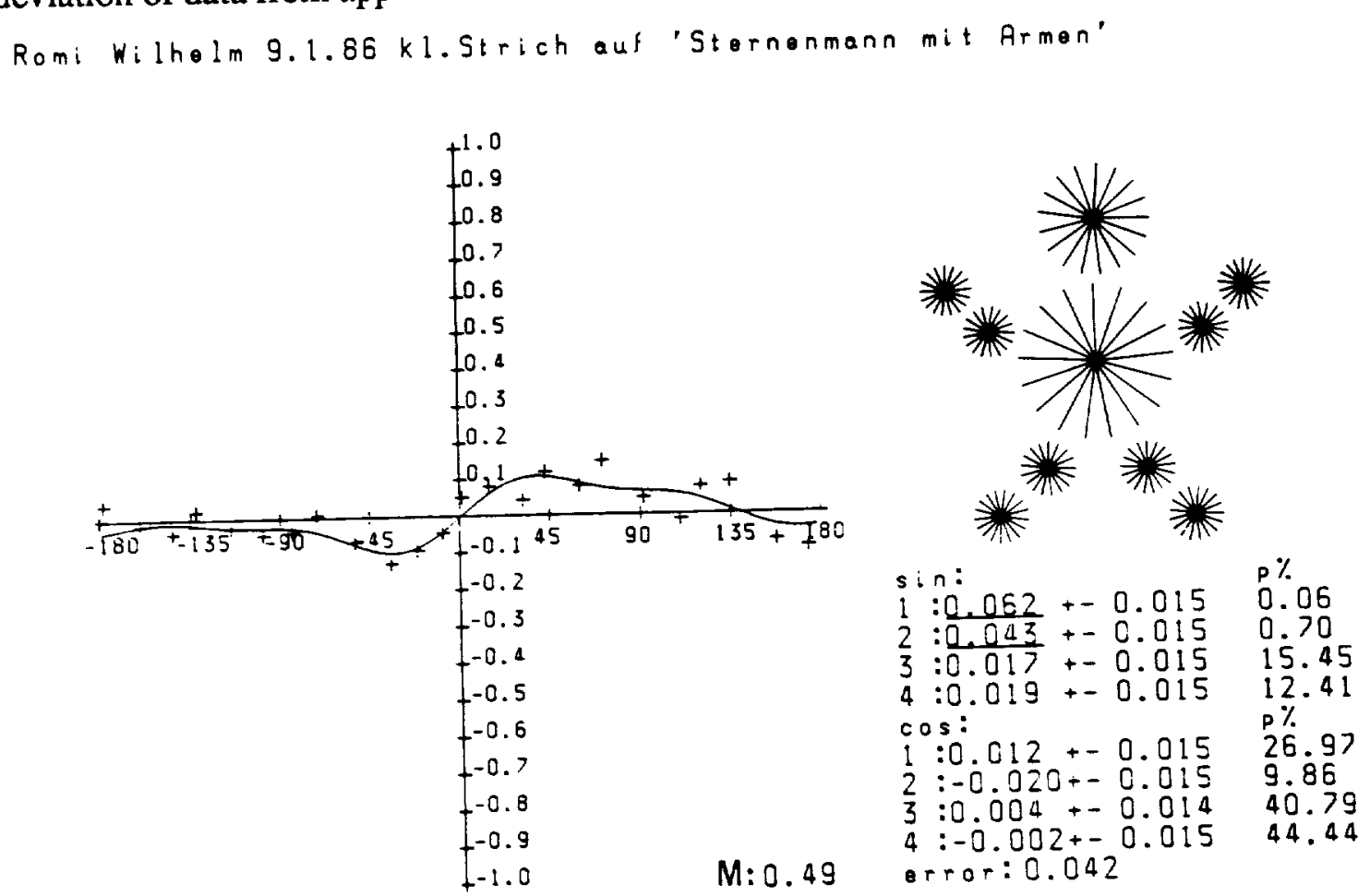

Figure 5.- Effect on SV of a figure which is composed of uniformly oriented luminous lines (star man). Procedures as in Figs. 1-3; symbols as in Fig. 4. Note that only the first and the second harmonic are significantly different from zero (two-tailed). 
R. Wilholm 3.12 .85 kl.Strich auf 'Rautenmann'
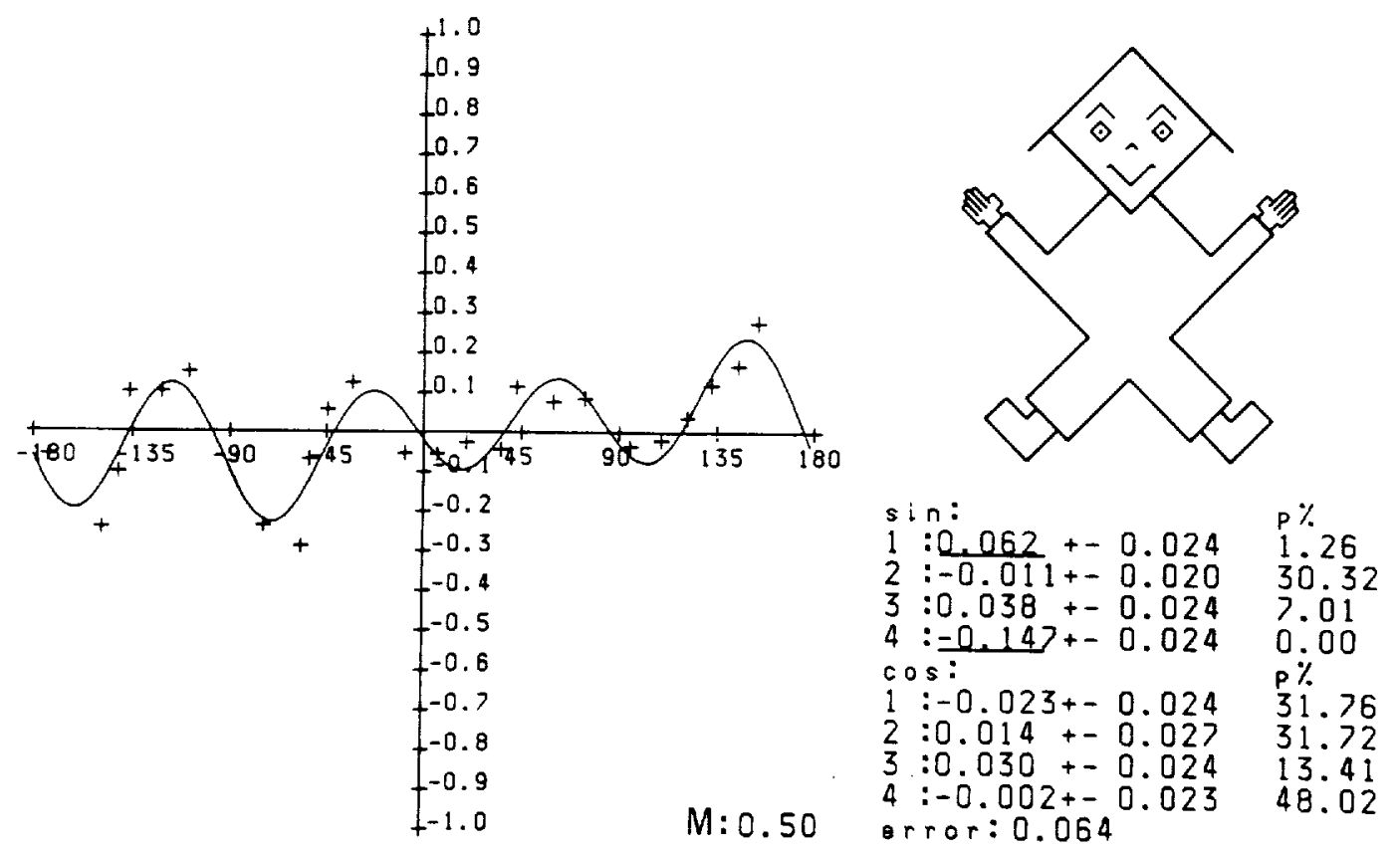

Figure 6.- Effect on SV of a figure which is composed of oblique luminous lines (diamond man). Note that the first and fourth harmonics are significantly (two-tailed) different from zero, but of different sign; that is, exactly (no cosines!) in counterphase at $\beta-\vartheta=0$. 


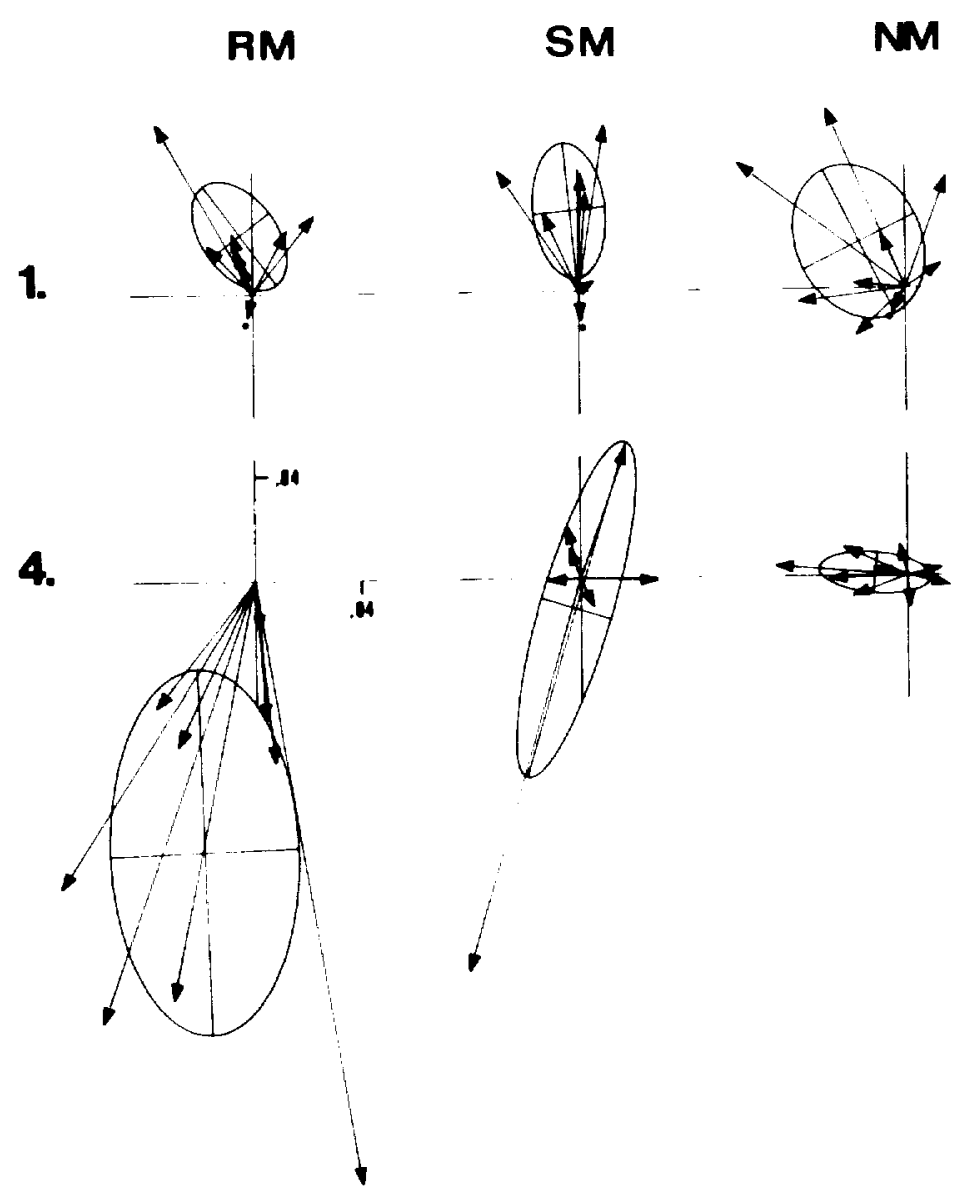

Figure 7.- First and fourth harmonics of experiments of Figs. 5 and 6 and in nine Ss. Location of arrowhead results from plotting sine amplitudes on ordinate and cosine amplitudes on abscissa (for scale see 4\% marks on fourth of RM). RM: diamond man of Fig. 6; SM: star man of Fig. 5; NM figure in the shape of SM, but composed of randomly oriented dashes ("needle man"). Ellipses: two-dimensional SD. Note similarity of 1. harmonics for all figures and in all Ss except one (dot under arrowhead), who evinces a negative 1. harmonic, that is, sees the polarity inverted. Furthermore, only RM engenders a significant fourth harmonic. 

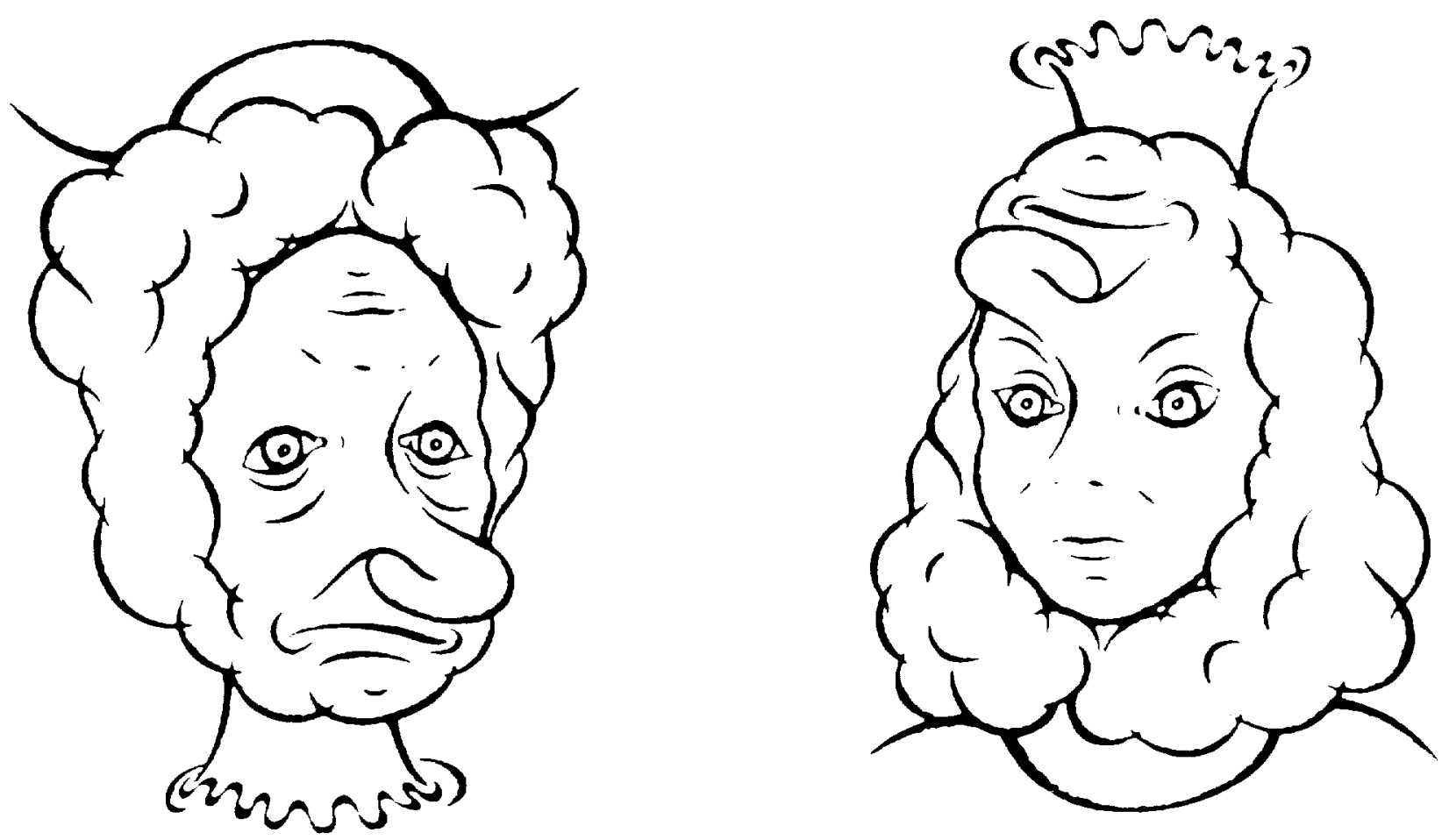

Figure 8.- The well-known ambiguous figure appearing as witch or princess upon inversion of long axis.

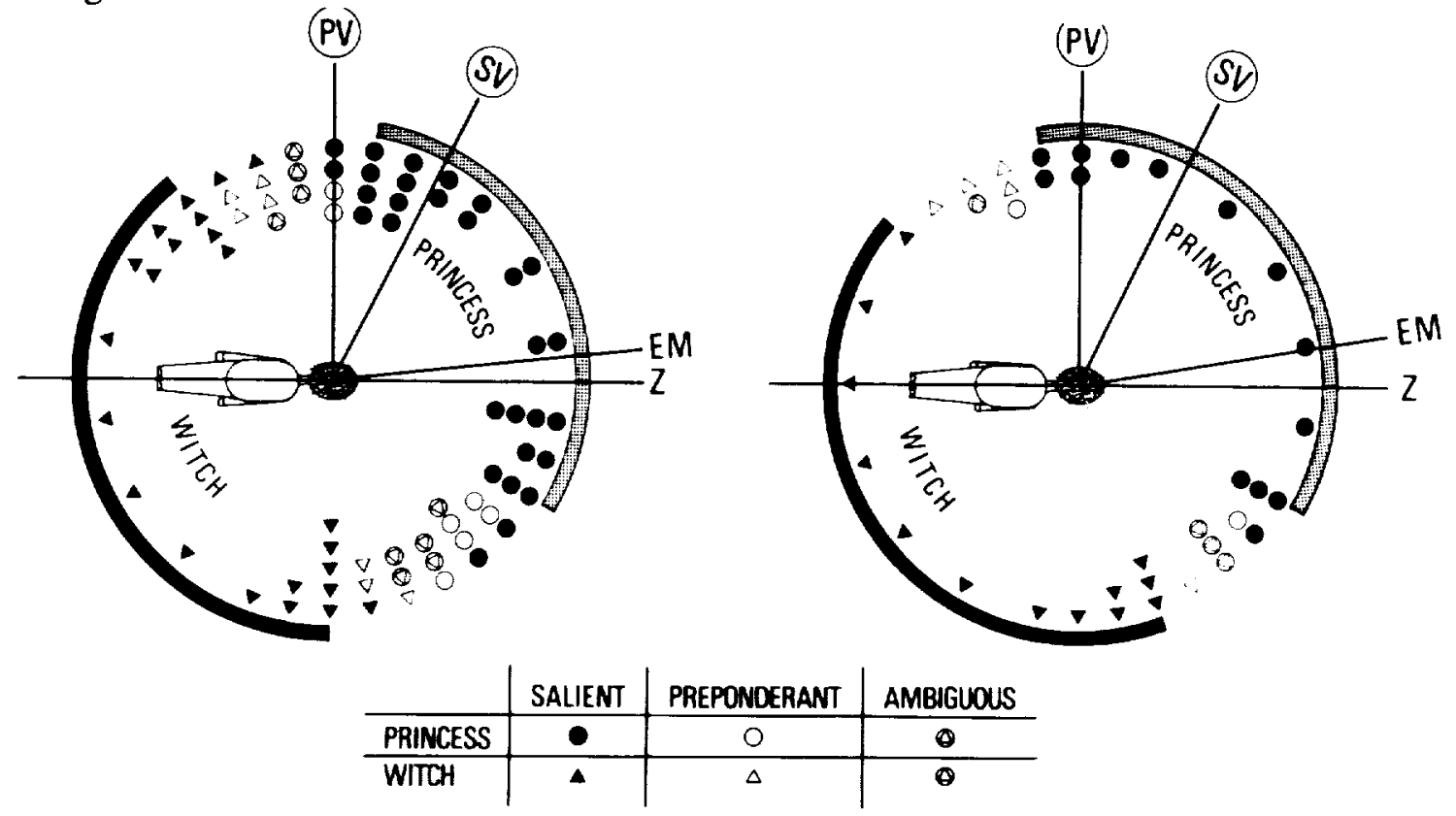

Figure 9.- To $S$ lying on the side, the princess is presented in various static orientations.

Direction of long (upright) axis of princess with respect to $S$ 's long axis (Z) is shown as direction of dot or triangle (like angle $\vartheta$ in Fig. 2). Type of symbol represents judgement of $S$ on how the figure appears to her when presented in that direction. One symbol stands for one presentation. More presentations were made in directions of critical transitions than in those of complete salience (exclusive distinctness). The latter are connected by black (witch exclusive) or grey (princess exclusive) circular segments. Note that the direction of the midline of saliency coincides neither with direction of the SV nor with that of the vertical retinal meridian (RM), nor with that of the physical vertical (PV). 